\title{
Three-Dimensional High-Resolution Black-Blood Magnetic Resonance Imaging for Detection of Arteritic Anterior Ischemic Optic Neuropathy in Patients With Giant Cell Arteritis
}

\author{
Nora N. Sommer, MD, * Karla M. Treitl, MD, *广 Eva Coppenrath, MD, * Hendrik Kooijman, PhD, $\neq$ \\ Claudia Dechant, MD, § Michael Czihal, MD,// Theresa M. Kolben, MD, $\mid$ Sebastian E. Beyer, MD,* \\ Wieland H. Sommer, MD, * and Tobias Saam, MD*\#
}

Objectives: Arteritic anterior ischemic optic neuropathy (A-AION) caused by inflammatory occlusion of the posterior ciliary arteries is the most common reason for irreversible vision loss in patients with giant cell arteritis. Atypical clinical presentation and negative funduscopy can delay systemic high-dose corticosteroid therapy to prevent impending permanent blindness and involvement of the contralateral eye.

The purpose of this study was to assess the diagnostic accuracy of 3-dimensional (3D) high-resolution T1-weighted black-blood magnetic resonance imaging (T1-BB-MRI) for the detection of posterior ciliary artery involvement in patients with giant cell arteritis and funduscopic A-AION.

Materials and Methods: After institutional review board approval and informed consent, 27 patients with suspected giant cell arteritis and vision disturbances were included in this monocentric prospective cohort study. Giant cell arteritis was diagnosed in 18 patients according to the diagnostic reference standard (6 men, 73.8 [69.0-78.0] years); 14 of those were positive for A-AION. Precontrast and postcontrast 3D T1-BB-MRI was performed in all 27 patients. Two radiologists separately assessed image quality and local fat suppression (4-point scale), visual contrast enhancement (3-point scale), and diagnostic confidence (5-point scale) regarding arteritic posterior ciliary artery involvement. Magnetic resonance imaging findings were assessed in comparison to funduscopy. Statistical analysis included accuracy parameters and interrater agreement.

Results: Sensitivity of 3D T1-BB-MRI was $92.9 \%$ (95\% confidence interval, $66.1 \%-99.8 \%$ ) and specificity was $92.3 \%$ (95\% confidence interval, $64.0 \%-$ $99.8 \%$ ) for detection of A-AION-positive patients. Image quality and local fat suppression were assessed with $3.2 \pm 0.8$ (median 3 ) and $3.8 \pm 0.5$ (median 4). Visual contrast enhancement with $2.3 \pm 0.8$ (median 3 ) and diagnostic confidence was rated at $4.7 \pm 0.5$ (median 5$)$. Interrater agreement was high $(\kappa=0.85$, $P<0.001$ ). Three-dimensional T1-BB-MRI displayed bilateral findings in $50 \%$ of the cases, whereas only unilateral A-AION was detected in funduscopy as a possible indication for the contralateral eye at risk.

Conclusions: Three-dimensional T1-BB-MRI allows accurate detection of arteritic posterior ciliary artery involvement in patients with A-AION. Further, 3D T1-BB-MRI seems to display arteritic involvement of the posterior ciliary arteries earlier than funduscopy and might, therefore, display "vision-at-risk"

Received for publication March 28, 2018; and accepted for publication, after revision, May 8, 2018

From the *Clinic and Polyclinic for Radiology, Ludwig-Maximilians-University Hospital; †German Center for Cardiovascular Disease Research, Munich; †Philips Healthcare, Hamburg; Divisions of §Rheumatology and Clinical Immunology, and ||Vascular Medicine, Medical Clinic and Policlinic IV, Ludwig-MaximiliansUniversity Hospital; $\uparrow$ Department for Gynecology and Obstetrics, Munich; and \#Radiologisches Zentrum Rosenheim, Rosenheim, Germany.

Disclosures: Hendrik Kooijman is employee of Philips Healthcare.

Conflicts of interest and sources of funding: none declared.

Correspondence to: Nora N. Sommer, MD, Clinic and Polyclinic for Radiology, Ludwig-Maximilians-University Hospital, Ziemssenstr. 1, 80336 Munich, Germany. E-mail: Nora.Sommer@med.uni-muenchen.de.

Copyright (c) 2018 Wolters Kluwer Health, Inc. All rights reserved.

ISSN: 0020-9996/18/5311-0698

DOI: $10.1097 /$ RLI.0000000000000500 in patients with visual impairment and suspected giant cell arteritis but unremarkable funduscopy.

Key Words: giant cell arteritis, arteritic anterior ischemic optic neuropathy, posterior ciliary arteries, 3D T1 black-blood imaging, T1-mVISTA, MP-RAGE, 3 T MRI

(Invest Radiol 2018;53: 698-704)

V ision loss occurs in approximately $20 \%$ of patients with giant cell arteritis (GCA) $)^{1,2}$ and is considered to be an ophthalmologic emergency requiring immediate diagnosis and early initiation of intravenous high-dose corticosteroid therapy to prevent impending permanent blindness and involvement of the contralateral eye. ${ }^{3-5}$

Arteritic anterior ischemic optic neuropathy (A-AION) is the most common reason for permanent vision impairment in patients with GCA. ${ }^{2,4,6}$ Arteritic anterior ischemic optic neuropathy is caused by arteritic ischemia of the anterior part of the optic nerve secondary to inflammatory occlusion of the posterior ciliary arteries. ${ }^{6-8}$ In the clinical context of GCA, a pale edematous optic nerve head is considered to be the funduscopic correlate of A-AION. ${ }^{7-9}$

However, diagnosing GCA and A-AION may be challenging: although the American College of Rheumatology has established criteria for the classification of $\mathrm{GCA},{ }^{10}$ validated diagnostic criteria are not available. Moreover, atypical clinical presentation of GCA is not unusual, with patients presenting with a normal erythrocyte sedimentation rate (ESR), ${ }^{11-13}$ or false-negative results of temporal artery biopsy (TAB) due to skip lesions. ${ }^{14-16}$ In addition, up to $21 \%$ of patients with biopsy-proven GCA present with isolated A-AION without systemic symptoms. ${ }^{17}$ Furthermore, arteritic posterior ischemic optic neuropathy (A-PION), a condition of ischemic occlusion of the pial vascular plexus supplied by collateral branches of the central retinal artery and the ophthalmic artery, is a rare cause of GCA-related blindness; however, it causes diagnostic difficulties, because no morphologic correlate can be seen in funduscopy. ${ }^{7-9}$ There are some promising results using diffusion-weighted imaging for detection of A-PION ${ }^{18,19}$; however, larger studies are needed to confirm this initial results.

Therefore, new diagnostic pathways should be explored. Blackblood magnetic resonance imaging (MRI) enables visualization of arteritic changes of vessels displaying circumferential thickening and strong contrast enhancement of the vessel wall. ${ }^{20-23}$ Until lately, conventional black-blood MRI based on double-inversion recovery techniques was restricted due to 2-dimensional (2D) acquisition mode, low spatial resolution, small coverage, and time concerns. ${ }^{20,24,25} \mathrm{We}$ recently developed a 3-dimensional (3D) T1-weighted (T1) blackblood (BB) turbo spin echo (TSE) sequence with variable flip-angle refocusing pulses and submillimeter isotropic resolution (3D T1-BB$\mathrm{MRI})$, allowing imaging of small vessels and image-reconstruction in all 3 planes. Three-dimensional T1-BB-MRI has been already proved to detect thoracic large vessel vasculitis ${ }^{26}$ and deep vein thrombosis. ${ }^{27}$ 
The purpose of this study was to assess the diagnostic accuracy of 3D T1-BB-MRI for the detection of posterior ciliary artery involvement in patients with GCA and funduscopic A-AION.

\section{MATERIALS AND METHODS}

\section{Study Design and Study Population}

The study was designed as a prospective monocentric cohort study. Institutional review board approval was given and written informed consent obtained from all patients before image acquisition. The study protocol followed the principles of the Declaration of Helsinki. All imaging procedures were performed for clinical reasons. Patients were recruited between September 2013 and April 2017.

\section{Inclusion Criteria}

Patients 50 years and older with visual symptoms (including amaurosis fugax, diplopia, rapid visual loss, blindness) and their first episode of suspected GCA were included in the study. After admission, all patients received (1) a complete MRI protocol (mentioned below) and (2) a funduscopy of both eyes.

\section{Diagnostic Reference Standard for the Diagnosis of GCA}

A definitive diagnosis or exclusion of GCA was determined for each patient according the diagnostic reference standard described subsequently: all patients suspected of GCA underwent laboratory testing (blood count, c-reactive protein, ESR) and a standardized color duplex sonography (CDS) of the superficial temporal arteries. A sonographer with more than 8 years of experience performed CDS using a GE LOGIC E9 ultrasound unit (GE Healthcare, Munich, Germany) with a 5- to 16-MHz broadband linear transducer. A hypoechogenic circumferential wall thickening (halo) in any segment of the temporal arteries was considered a positive CDS finding for GCA. ${ }^{28,29}$ The diagnosis of GCA was finally established in an interdisciplinary consensus decision by experienced rheumatologists and angiologists by fulfillment of at least 3 of the American College of Rheumatology classification criteria for cranial GCA, ${ }^{10}$ in the presence of the typical CDS findings mentioned previously or by a positive $\mathrm{TAB}$, together with typical clinical signs of GCA.

\section{Funduscopic Examination}

All patients underwent an ophthalmologic council including funduscopy. Funduscopic findings were reviewed by experienced ophthalmologists to diagnose or exclude A-AION according to the following criteria: if funduscopy revealed a pale edematous optic disc, A-AION was considered to be the cause, whereas a hyperemic edema of the optic disc and a small cup with a crowded optic disc contralaterally were referred to be a cause of a nonarteritic atherosclerotic AION (NA-AION). ${ }^{6-8,30}$

\section{Exclusion Criteria}

Patients were excluded when one of the following criteria was present: (1) definitive diagnosis or exclusion of GCA was not possible based on the reference standard; (2) GCA suspected, but patients had corticosteroid therapy for more than 14 days before admission, as vasculitic changes were shown to diminish between 6 to 14 days and decrease significantly afterwards ${ }^{22}$; and (3) nondiagnostic MRI due to an incomplete protocol and insufficient image quality.

\section{MRI Protocol}

All patients were scanned with a 3.0 T scanner (Ingenia; Philips Healthcare, Best, the Netherlands) using a standard commercial 15-channel head coil.
Three-dimensional T1-BB-MRI covering the whole brain was performed before and after application of $0.2 \mathrm{~mL} / \mathrm{kg}$ body weight of gadoteric acid (DOTAREM $0.5 \mathrm{mmol} / \mathrm{mL}$; Guerbet, Roissy, France). The first postcontrast scan was started 5 minutes after injection. Detailed scan parameters are listed in Table 1.

\section{Image Analysis}

A member of the study group, who did not act as a reader and was aware of the confirmed clinical diagnosis, selected the images.

Two radiologists, blinded to the clinical diagnosis with more than 3 years of experience in MRI, independently analyzed the images on a picture archiving and communication system (Syngo; Siemens AG Healthcare, Erlangen, Germany). Although the sequence covered the whole brain, readers were advised to assess the orbital cavity exclusively. Images of patients with and without GCA were provided in a random order for both readers. Precontrast and postcontrast images were reconstructed in all 3 planes with a slice thickness of $1.5 \mathrm{~mm}$. Right and left orbital cavities were assessed separately.

Image quality and fat suppression of the orbital cavity were rated as follows: 1, diagnosis strongly impaired; 2 , adequate (major artifacts); 3 , good (minor artifacts); 4, excellent (no artifacts).

Readers had to assess whether or not arteritic involvement of the posterior ciliary arteries was present with typical appearance (circumferential vessel wall thickening and strong contrast enhancement of the vessel wall ${ }^{20-23}$ ) and to assess the diagnostic confidence (DC) for the presence of vasculitis (1, unlikely; 2, vague; 3, likely; 4, high; 5 , very high). A DC of 4 and 5 were considered diagnostic. Furthermore, assessment of visual contrast enhancement (VCE) of the posterior ciliary arteries was rated as follows: 0, none; 1, minimal; 2 , moderate; and 3, strong contrast enhancement. Reading was performed separately. A consensus reading was performed in the case of any differences.

TABLE 1. Parameters of 3D T1-BB-MRI

\section{D T1-mVISTA}

\begin{tabular}{lc}
\hline Sequence type & 3D T1 TSE \\
TR, ms & 700 \\
TE, ms & 24 \\
Fat suppression & SPIR \\
SENSE factor (AP)/(RL) & $1.7 / 2.5$ \\
Echo spacing/ETL & $4.4 / 268$ \\
Echo pulse angle, degree & Variable; minimum, 50 degrees; \\
& maximum 120 degrees \\
NSA & 2 \\
Scan FOV, mm ${ }^{3}$ & $200 \times 251 \times 160$ \\
Recon matrix & 640 \\
No. slices & 427 \\
Voxel size, mm & 3 \\
Recon voxel size, mm & 3 \\
Scan time, min & $0.75 \times 0.75 \times 0.75$ \\
Flow compensation & $0.39 \times 0.39 \times 0.35$ \\
Slice orientation & $4: 43$ \\
\hline
\end{tabular}

3D indicates 3-dimensional; T1-BB-MRI, T1-weighted black-blood magnetic resonance imaging; AP, anterior-posterior; ETL, echo train length; FOV, field of view; NSA, number of signal averages; RL, right-left; SENSE, parallel acquisition technique; SPIR, spectral presaturation with inversion recovery; T1, T1-weighted; TE, echo time; TR, repetition time; TSE, turbo spin echo; T1-mVISTA, T1weighted modified volumetric isotropic TSE acquisition. 


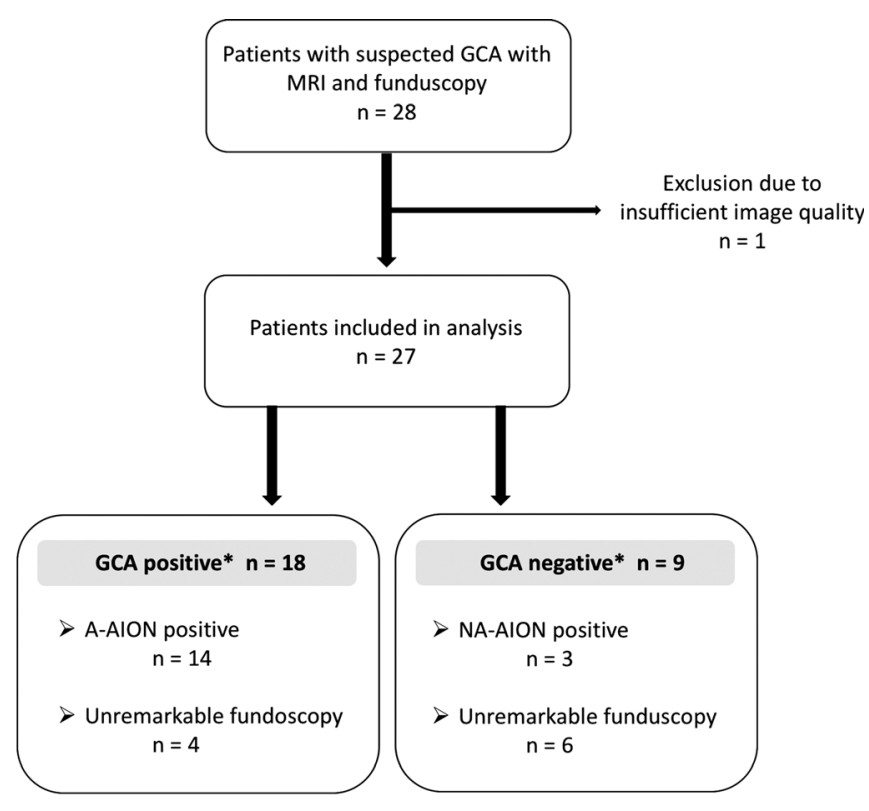

*According to the diagnostic reference standard for GCA of the study protocol

FIGURE 1. Flowchart displaying the selection of patients. A-AION, arteritic anterior ischemic optic neuropathy; GCA, giant cell arteritis; MRI, magnetic resonance imaging; NA-AION, nonarteritic anterior ischemic optic neuropathy.

\section{Statistical Analysis}

We performed all statistical analyses using SPSS 23 (IBM, Armonk, NY).

All metric and normally distributed variables are reported as mean \pm standard deviation (SD); nonnormally distributed variables are presented as median with first-third quartiles (IQR). Ordinal variables are reported using both median plus IQR and mean \pm SD. Categorical variables are presented as frequency and percentage.

Normal distribution was assessed by the Kolmogorov-Smirnov test. The $\chi^{2}$ test was used for binomial variables. The $t$ test and MannWhitney $U$ test were used for continuous normally and nonnormally distributed variables, respectively. Mann-Whitney $U$ test was used for 2 sample comparisons of ordinal variables. Two-sided $P$ values were determined, and values below 0.05 were considered significant.

Interreader variability of the qualitative parameter "MRI changes" was assessed by Cohen $\kappa$.

\section{RESULTS}

\section{Patient Population}

A total of 28 patients with visual symptoms suspected for GCA received MRI. One patient was excluded due to motion artifacts. Eighteen of the 27 patients received the final diagnosis GCA (Fig. 1).
Other diagnoses were found as cause of the symptoms in the remaining nine patients: 3 patients were diagnosed with NA-AION of the right eye $(n=2)$ and the left eye $(n=1), 1$ with a small subacute thromboembolic infarction of the visual cortex, and 5 with nonspecific headache and other causes of visual impairment.

Nine of the 27 patients received TAB in addition to ultrasound to establish the diagnosis.

Patients with clinically confirmed GCA tended to be older than patients without GCA, although this difference was not significant (Table 2). There were no significant differences between the cohorts regarding sex and onset time of application of systemic corticosteroids, if administered (Table 2).

\section{Image Quality of 3D T1-BB-MRI}

All images $(n=27)$ had diagnostic quality with a median of 3 (IQR 3-4; mean \pm SD, $3.3 \pm 0.8$ ) for the right orbital cavity and a median of 3 (IQR 2-4; mean \pm SD, $3.1 \pm 0.8$ ) for the left orbital cavity on a 4-point scale without significant difference regarding right or left side ( $U$ value, $322.5 ; Z$ score, $0.718 ; P=0.471$ ). Image quality was assessed to be good to excellent in $77.8 \%$ of the cases. Artifacts were seen at the junction of the maxillary sinus and the orbital cavity, but image quality was not significantly impaired.

Fat suppression was rated with a median of 4 (IQR 4-4; mean $\pm \mathrm{SD}, 3.8 \pm 0.5$ ) for the right orbital cavity and a median of 4 (IQR 4-4; mean $\pm \mathrm{SD}, 3.8 \pm 0.6$ ) for the left orbital cavity on a 4-point scale without significant differences between right and left side ( $U$ value, 362.5 ; $Z$ score, $0.026 ; P=0.976$ ). Local fat suppression was rated to be good to excellent in $96.3 \%$ of the cases.

\section{Findings in Funduscopy}

As mentioned previously, 3 patients without GCA were diagnosed with atherosclerotic NA-AION. Funduscopy was unremarkable in the other 6 non-GCA patients.

Fourteen $(77.8 \%)$ of the 18 patients diagnosed with GCA were rated positive for A-AION. The latter was detected unilaterally in 8 patients ( 6 right eyes, 2 left eyes) and bilaterally in 6 patients. Fundus appearance was unremarkable in the 4 other patients with GCA.

\section{Findings in 3D T1-BB-MRI}

None of the patients without GCA displayed contrast enhancement of vessels in the orbital cavity (Fig. 2).

Fourteen of the 18 patients with GCA displayed a strong and blurry contrast enhancement aside the optic nerve and the adjacent orbital fat following the course of the posterior ciliary arteries (Fig. 2). Bilateral enhancement was reported in 13 cases (Fig. 3); unilateral enhancement of the right side was detected in 1 case.

In the case of contrast enhancement, VCE was rated with a median of 2 (IQR 2-3; mean $\pm \mathrm{SD}, 2.3 \pm 0.7$ ) for the right side and a median of 3 (IQR 2-3; mean $\pm \mathrm{SD}, 2.4 \pm 0.9$ ) for the left side on a 3-point scale with a DC regarding arteritic involvement with a median of 5 (IQR 4-5; mean $\pm \mathrm{SD}, 4.6 \pm 0.5$ ) and a median of 5 (IQR 5-5; mean $\pm \mathrm{SD}, 4.7 \pm 0.6$ ) on a 5-point scale for the right and the left side,

TABLE 2. Patient Characteristics

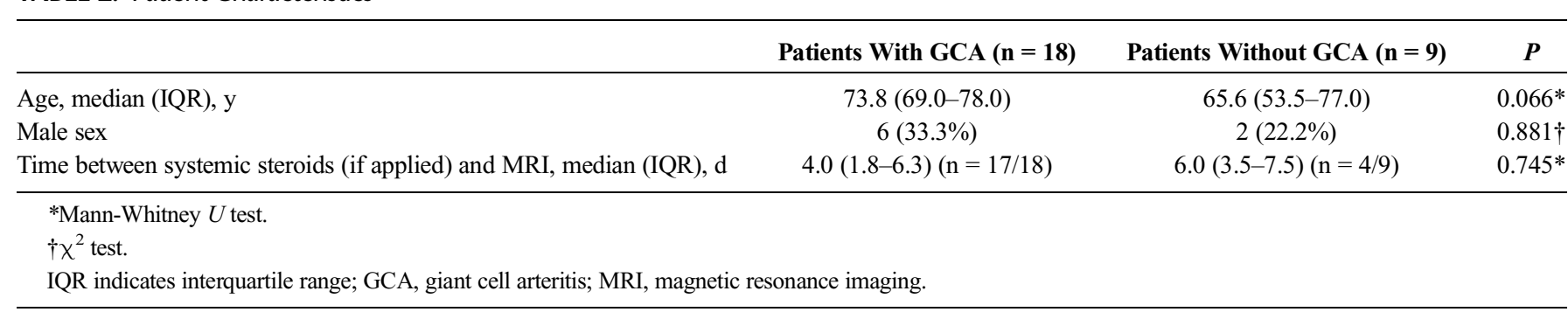



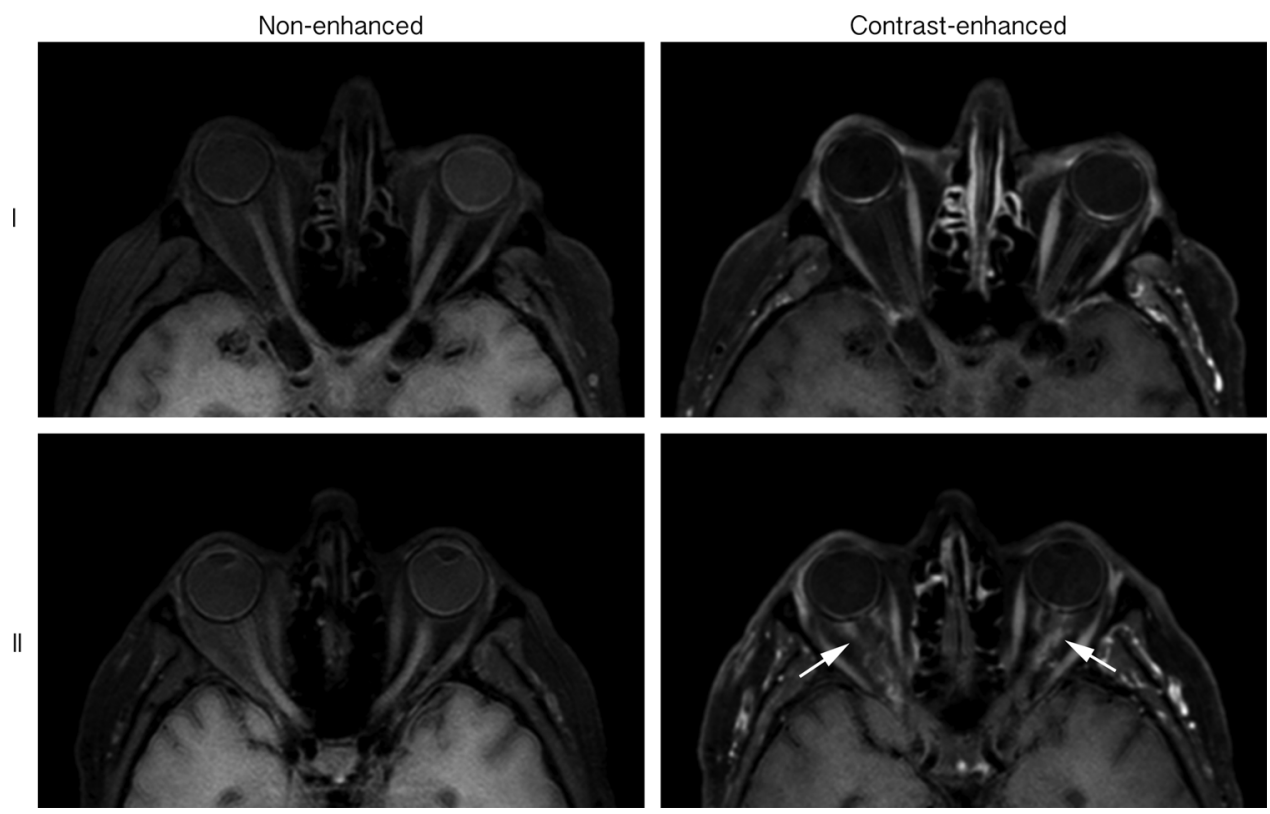

FIGURE 2. The figure displays images of nonenhanced and contrast-enhanced fat suppressed 3D T1-BB-MRI in axial reconstruction of 2 different patients: patient 1 (upper row) was an 81-year-old man without GCA according to the diagnostic reference standard and NA-AION of the right eye; MRI did not show any contrast enhancement of the small vessels adjacent to the optic nerve. Patient 2 (lower row) was a 72-year-old man with TAB-positive GCA and bilateral A-AION detected by funduscopy; MRI displayed strong patchy contrast enhancement following the course of the posterior ciliary arteries aside both optic nerves and the adjacent orbital fat (laterally marked by arrows); high-dose corticosteroid therapy was started 5 days before MRI acquisition. A-AION, arteritic anterior ischemic optic neuropathy; GCA, giant cell arteritis; NA-AION, nonarteritic anterior ischemic optic neuropathy; TAB, temporal artery biopsy; MRI, magnetic resonance imaging; 3D T1-BB-MRI, 3-dimensional T1-weighted black-blood magnetic resonance imaging.

respectively. No significant differences were detected between right and left side regarding VCE ( $U$ value, $87.0 ; Z$ score, $-0.482 ; P=0.631$ ) or DC ( $U$ value, $86.5 ; Z$ score, $-0.505 ; P=0.610)$, respectively.

\section{Findings of 3D T1-BB-MRI Compared With Funduscopic Findings in GCA Patients}

Thirteen of the 18 GCA patients displayed both positive findings in funduscopy regarding A-AION and in MRI regarding arteritic involvement of the posterior ciliary arteries. Three patients did not show any changes either in funduscopy or in MRI. One patient with unilateral funduscopic A-AION of the right eye was not detected by MRI. Another patient negative for A-AION in funduscopy displayed bilaterally strong contrast enhancement in MRI indistinguishable to the other 13 patients who were rated positive both by funduscopy and MRI (Table 3 and Fig. 3, patient 4). When comparing MRI changes and funduscopy as clinical reference standard in any eye, MRI showed a sensitivity $92.9 \%$ (95\% confidence interval [CI], 66.1\%-99.8\%) and a specificity of $92.3 \%(95 \%$ CI, 64.0\%-99.8\%). Positive predictive value was $92.9 \%(95 \% \mathrm{CI}, 66.3 \%-98.9 \%)$, and negative predictive value was $92.3 \%$ (95\% CI, 64.3\%-98.8\%).

In general, MRI displayed bilateral involvement in 12 of the 13 cases, whereas funduscopy revealed bilateral A-AION in only 6 of the 13 cases (Fig. 3). Both MRI and funduscopy detected only affection of the right side in the 1 remaining case.

\section{Reproducibility}

There was excellent interreader agreement between both readers in the assessment of MRI changes (Table 4).

\section{DISCUSSION}

This prospective single-center cohort study provides evidence that high-resolution contrast-enhanced 3D black-blood MRI allows accurate diagnosis of ophthalmic involvement in patients with GCA Interreader agreement was excellent $(\kappa=0.852, P<0.001)$, implying that 3D black-blood MRI of the orbital cavity for detection of arteritic involvement in patients with GCA is reproducible.

Our findings are in line with previous case reports mentioning contrast enhancement of the optic nerve sheath and the adjacent orbital fat in patients in with biopsy-proven GCA and corresponding unilat$\mathrm{eral}^{31}$ and bilateral A-AION in funduscopy, ${ }^{32-34}$ respectively.

Moreover, 3D T1-BB-MRI displayed bilateral affection in 12 cases, whereas funduscopy revealed bilateral A-AION in only 6 of these cases. These findings are also in line with 2 other case reports: both patients were presenting with unilateral A-AION, but bilateral nodular optic nerve sheath enhancement in MRI. The ESR and c-reactive protein were normal; however, TAB revealed GCA ${ }^{35,36}$ Furthermore, other authors described a case of an 83-year-old woman with rapid progression of bilateral vision loss, but unremarkable fundus appearance and normal ESR. The MRI displayed bilateral perineural optic nerve enhancement, and TAB was consistent with GCA. ${ }^{37}$ The authors stated that A-PION might have played a role in this patient.

We also encountered 1 patient in our study with bilateral unremarkable funduscopy, but bilateral contrast enhancement in MRI indistinguishable from the other 13 patients who were rated positive both by funduscopy and MRI (Fig. 3, patient 4).

Arteritic anterior ischemic optic neuropathy is the morphologic correlate displayed by funduscopy when ischemia has occurred with an irreversible impairment of vision. Considering our observations and the case reports described, MRI seems to depict arteritic ophthalmic involvement before critic ischemia has developed and might, therefore, reveal "vision at risk" before being detectable by funduscopy.

Although the other authors described contrast enhancement of the optic nerve sheath, considering the known pathophysiology of A-AION, it seems likely that the patchy contrast enhancement adjacent to the optic nerve displays rather inflammation of the posterior ciliary 

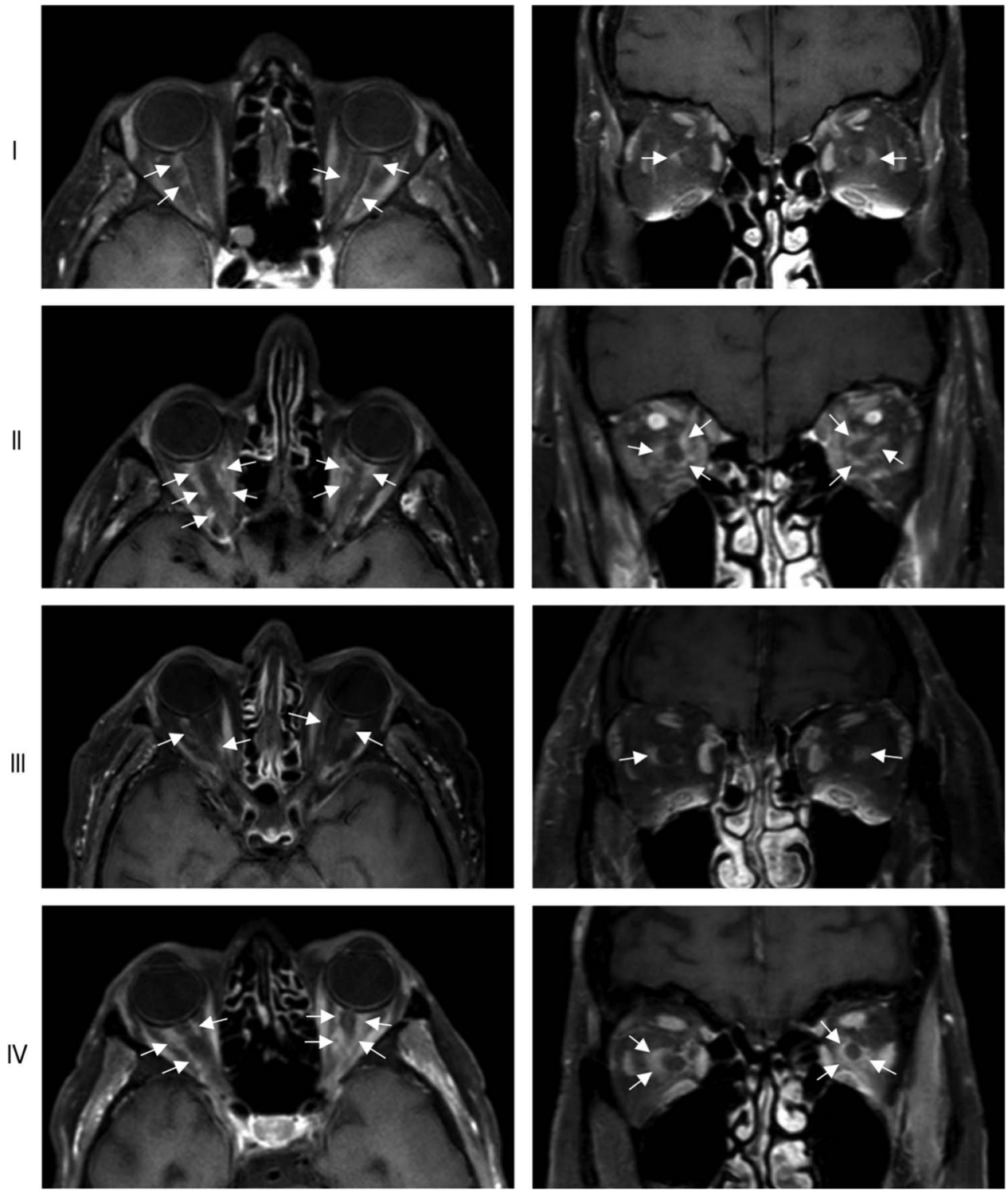

A

B

FIGURE 3. This figure displays images of axial $(A)$ and coronal $(B)$ reconstructions of contrast-enhanced fat suppressed 3D T1-BB-MRI of 4 different patients: patient 1: 68-year-old man with GCA and bilateral A-AION as diagnosed by the reference standard; contrast-enhanced MRI displays strong blurry and patchy enhancement adjacent to the optic nerve bilaterally after the course of the posterior ciliary arteries (arrows), whereas the sheaths of the optic nerve do not enhance. Susceptibility artifacts can be seen at the junction of the maxillary sinus and the orbit in the coronal reconstruction, but without overall diagnostic impairment; high-dose corticosteroid therapy was started 14 days before MRI acquisition. Patient 2: 52-year-old man with GCA and A-AION of the right eye with unremarkable funduscopy of the left eye; however, MRI displayed blurry contrast enhancement of the posterior ciliary arteries aside the optic nerve of both eyes (arrows) without obvious differences between both sides. The prominent hyperintense superior ophthalmic vein in both sides visible in the coronal image reconstruction due to ultra-low flow was also notable, however, no other pathologies, such as retinal venous occlusion, were detected by funduscopy; high-dose corticosteroid therapy was started 1 day before MRI acquisition. Patient 3: 80-year-old woman with GCA and unilateral A-AION of the left eye detected by funduscopy; MRI again displayed bilateral patchy contrast enhancement aside both optic nerves (arrows). Artifacts at the junction of the maxillary sinus and the orbit can be seen on both sides, but without significant diagnostic constraints; high-dose corticosteroid therapy was started 2 days before MRI acquisition. Patient 4: 94-year-old woman with confirmed GCA, but with unremarkable funduscopy negative for A-AION, but visual disturbances of both eyes; indistinguishable to all other cases shown above, MRI displays strong, patchy contrast enhancement along the optic nerves and the adjacent orbital fat in the course of the posterior ciliary arteries (arrows); high-dose corticosteroid therapy was started 2 days before MRI acquisition. A-AION, arteritic anterior ischemic optic neuropathy; GCA, giant cell arteritis; TAB, temporal artery biopsy; MRI, magnetic resonance imaging; 3D T1-BB-MRI, 3-dimensional T1-weighted black-blood magnetic resonance imaging.

arteries than being correlate of an inflammation of the nerve sheath. This finding has probably to be attributed to the fact that standard 2D T1 SE sequences were used without specific blood flow suppression techniques and with a lower resolution and higher slice thickness, therefore, lacking the differentiation of anatomic structures aside the optic nerve. This hypothesis is supported by Morgenstern et al, ${ }^{34}$ who found giant cell infiltration of the perineural vasculature in a biopsy of a patient presenting with bilateral 
enhancement of the optic nerve sheath and bilateral A-AION. Moreover, a breakdown of the blood-optic nerve barrier ${ }^{37}$ or neovascularization, ${ }^{38}$ as suggested by other authors, seems unlikely considering the known pathomechanism of A-AION.

A systematic analysis performed by Geiger et $\mathrm{al}^{39}$ to investigate the diagnostic impact of MRI for the detection of ophthalmic involvement in patients with GCA could not establish any correlation between funduscopy, visual symptoms, and MRI. The authors stated that this was probably related to the size of the arteries, as they could not detect the posterior ciliary arteries with their proposed protocol of 2D SE sequences. Furthermore, arteritic changes of the ophthalmic artery, as observed in their study, are not causative for A-AION.

In our study using 3D T1-BB-MRI with a higher spatial resolution and a reconstructed slice thickness of $1.5 \mathrm{~mm}$, the course of the posterior ciliary arteries aside the optic nerve could be clearly followed and there were typical signs for vasculitis, such as circumferential wall thickening, strong contrast enhancement, and inflammation of the adjacent soft tissue. ${ }^{20,40}$

A substantial limitation of our study is the small sample size. However, GCA is a rare disease and we needed to apply strong inclusion criteria, such as first-time manifestation of GCA and coexistence of A-AION. A prospective multicenter study is necessary to further substantiate these preliminary findings.

Further, the sequence covered the entire head and readers had access to all image reconstructions. Although readers were advised to assess the orbital cavity exclusively, we cannot rule out that other vessels prone to vasculitic involvement, like the temporal artery, were also looked at and might have influenced the decision making.

Moreover, because biopsy of the posterior ciliary arteries in any patient would not have been justifiable, we can only assume that MR displays arteritic involvement of the posterior ciliary arteries. Similarly, there is no clinically applicable criterion standard for diagnosing A-PION. As we did not perform a biopsy and A-PION cannot be proved by funduscopy, we can only hypothesize that bilateral contrast enhancement is indicating the contralateral eye at risk. This is also supported by the fact that we did not detect contrast enhancement in any of the patients without GCA.

Lastly, we did not compare 3D T1-BB-MRI to conventional 2D T1 SE or other sequences.

\section{CONCLUSIONS}

Our findings demonstrate that 3D T1-BB-MRI allows accurate diagnosis of arteritic involvement of the posterior ciliary arteries in patients with GCA and funduscopic A-AION. Moreover, our observations suggest that MRI can depict ophthalmic involvement much earlier than funduscopy and might, hence, indicate "vision at risk" before irreversible vision loss occurs.

TABLE 3. Performance of 3D T1-BB-MRI Regarding Detection of Vasculitic Changes of the Posterior Ciliary Arteries Compared With Funduscopy Detecting A-AION in Any Eye in All Patients $(n=27)$

\begin{tabular}{lcc}
\hline & A-AION Positive & A-AION Negative \\
\hline MRI positive & $13 / 48.2 \%$ & $1 / 3.7 \%$ \\
MRI negative & $1 / 3.7 \%$ & $12 / 44.4 \%$ \\
\hline
\end{tabular}

\footnotetext{
$\chi^{2}$ test: $P<0.001$
}

A-AION indicates arteritic anterior ischemic optic neuropathy; MRI, magnetic resonance imaging; 3D T1-BB-MRI, 3-dimensional T1-weighted black-blood magnetic resonance imaging.
TABLE 4. Interrater Variability Regarding Detection of Vasculitic Changes of the Posterior Ciliary Arteries in 3D T1-BB-MRI

\begin{tabular}{lccc}
\hline & $\mathbf{n}$ & Cohen $\boldsymbol{\kappa}$ & $\boldsymbol{P}$ \\
\hline Vasculitic changes in any eye & 27 & 0.852 & $<0.001$ \\
Vasculitic changes right eye & 27 & 0.852 & $<0.001$ \\
Vasculitic changes left eye & 27 & 0.926 & $<0.001$ \\
\hline
\end{tabular}

3DT1-BB-MRI indicates 3-dimensional T1-weighted black-blood magnetic resonance imaging.

Therefore, 3D T1-BB-MRI might provide diagnostic support, especially in clinically ambiguous cases when vision disturbances due to GCA are suspected, but patients present with normal inflammatory blood parameters and unremarkable fundus appearance.

\section{REFERENCES}

1. Singh AG, Kermani TA, Crowson CS, et al. Visual manifestations in giant cell arteritis: trend over 5 decades in a population-based cohort. $J$ Rheumatol. 2015;42:309-315.

2. Liu GT, Glaser JS, Schatz NJ, et al. Visual morbidity in giant cell arteritis. Clinica characteristics and prognosis for vision. Ophthalmology. 1994;101:1779-1785.

3. Hayreh SS. Anterior ischemic optic neuropathy. Clin Neurosci. 1997;4:251-263.

4. González-Gay MA, García-Porrúa C, Llorca J, et al. Visual manifestations of giant cell arteritis. Trends and clinical spectrum in 161 patients. Medicine (Baltimore). 2000;79:283-292.

5. Gonzalez-Gay MA, Martinez-Dubois C, Agudo M, et al. Giant cell arteritis: epidemiology, diagnosis, and management. Curr Rheumatol Rep. 2010;12:436-442.

6. Soriano A, Muratore F, Pipitone N, et al. Visual loss and other cranial ischaemic complications in giant cell arteritis. Nat Rev Rheumatol. 2017;13:476-484.

7. Hayreh SS. Ischemic optic neuropathy. Prog Retin Eye Res. 2009;28:34-62.

8. Biousse V, Newman NJ. Ischemic optic neuropathies. $N$ Engl J Med. 2015;372: 2428-2436.

9. Biousse V, Newman NJ. Diagnosis and clinical features of common optic neuropathies. Lancet Neurol. 2016;15:1355-1367.

10. Hunder GG, Bloch DA, Michel BA, et al. The American College of Rheumatology 1990 criteria for the classification of giant cell arteritis. Arthritis Rheum. 1990;33:1122-1128.

11. Kermani TA, Schmidt J, Crowson CS, et al. Utility of erythrocyte sedimentation rate and C-reactive protein for the diagnosis of giant cell arteritis. Semin Arthritis Rheum. 2012;41:866-871

12. Parikh M, Miller NR, Lee AG, et al. Prevalence of a normal C-reactive protein with an elevated erythrocyte sedimentation rate in biopsy-proven giant cell arteritis. Ophthalmology. 2006;113:1842-1845.

13. Yoeruek E, Szurman P, Tatar O, et al. Anterior ischemic optic neuropathy due to giant cell arteritis with normal inflammatory markers. Graefes Arch Clin Exp Ophthalmol. 2008;246:913-915.

14. Goslin BJ, Chung MH. Temporal artery biopsy as a means of diagnosing giant cell arteritis: is there over-utilization? Am Surg. 2011;77:1158-1160.

15. Durling B, Toren A, Patel V, et al. Incidence of discordant temporal artery biopsy in the diagnosis of giant cell arteritis. Can J Ophthalmol. 2014;49:157-161.

16. Poller DN, van Wyk Q, Jeffrey MJ. The importance of skip lesions in temporal arteritis. J Clin Pathol. 2000;53:137-139.

17. Hayreh SS, Podhajsky PA, Zimmerman B. Occult giant cell arteritis: ocular manifestations. Am J Ophthalmol. 1998;125:521-526.

18. Bender B, Heine C, Danz S, et al. Diffusion restriction of the optic nerve in patients with acute visual deficit. J Magn Reson Imaging. 2014;40:334-340.

19. Quddus A, Lawlor M, Siddiqui A, et al. Using diffusion-weighted magnetic resonance imaging to confirm a diagnosis of posterior ischaemic optic neuropathy: two case reports and literature review. Neuroophthalmology. 2015;39:161-165.

20. Choi YJ, Jung SC, Lee DH. Vessel wall imaging of the intracranial and cervical carotid arteries. J stroke. 2015;17:238-255.

21. Schwarz F, Strobl FF, Cyran CC, et al. Reproducibility and differentiation of cervical arteriopathies using in vivo high-resolution black-blood MRI at $3 \mathrm{~T}$. Neuroradiology. 2016;58:569-576.

22. Klink T, Geiger J, Both M, et al. Giant cell arteritis: diagnostic accuracy of MR imaging of superficial cranial arteries in initial diagnosis-results from a multicenter trial. Radiology. 2014;273:844-852. 
23. Pfefferkorn T, Schuller U, Cyran C, et al. Giant cell arteritis of the basal cerebral arteries: correlation of MRI, DSA, and histopathology. Neurology. 2010;74: 1651-1653.

24. Dieleman N, Van Der Kolk AG, Zwanenburg JJ, et al. Imaging intracranial vessel wall pathology with magnetic resonance imaging current prospects and future directions. Circulation. 2014;130:192-201.

25. Saam T, Raya JG, Cyran CC, et al. High resolution carotid black-blood 3 T MR with parallel imaging and dedicated 4-channel surface coils. J Cardiovasc Magn Reson. 2009;11:41

26. Treitl KM, Maurus S, Sommer NN, et al. 3D-black-blood 3T-MRI for the diagnosis of thoracic large vessel vasculitis: a feasibility study. Eur Radiol. 2017; 27:2119-2128.

27. Treitl KM, Treitl M, Kooijman-Kurfuerst H, et al. Three-dimensional black-blood T1-weighted turbo spin-echo techniques for the diagnosis of deep vein thrombosis in comparison with contrast-enhanced magnetic resonance imaging: a pilot study. Invest Radiol. 2015;50:401-408.

28. Czihal M, Piller A, Schroettle A, et al. Impact of cranial and axillary/subclavian artery involvement by color duplex sonography on response to treatment in giant cell arteritis. J Vasc Surg. 2015;61:1285-1291.

29. Salvarani C, Cantini F, Hunder GG. Polymyalgia rheumatica and giant-cell arteritis. Lancet. 2008;372:234-245.

30. Hayreh SS, Podhajsky PA, Zimmerman B. Ocular manifestations of giant cell arteritis. Am J Ophthalmol. 1998;125:509-520.
31. D'Souza NM, Morgan ML, Almarzouqi SJ, et al. Magnetic resonance imaging findings in giant cell arteritis. Eye (Lond). 2016;30:758-762.

32. Osman SS, Ahem A. Value of MRI in diagnosis of giant cell arteritis. Neurol Asia. 2012;17:369-372

33. Morotti A, Padovani A, Liberini P. Bilateral optic perineuritis as the presenting feature of giant cell arteritis. BMJ Case Rep. 2013;pii:bcr2011007959.

34. Morgenstern KE, Ellis BD, Schochet SS, et al. Bilateral optic nerve sheath enhancement from giant cell arteritis. J Rheumatol. 2003;30:625-627.

35. Liu TYA, Miller NR. Giant cell arteritis presenting as unilateral anterior ischemic optic neuropathy associated with bilateral optic nerve sheath enhancement on magnetic resonance imaging. J Neuroophthalmol. 2015;35:360-363.

36. Kornberg MD, Ratchford JN, Subramaniam RM, et al. Giant cell arteritis mimicking infiltrative leptomeningeal disease of the optic nerves. BMJ Case Rep. 2015 pii:bcr2014209160.

37. Liu KC, Chesnutt DA. Perineural optic nerve enhancement on magnetic resonance imaging in giant cell arteritis. $J$ Neuroophthalmol. 2013;33:279-281

38. Garcia-Porrua C, Pego-Reigosa R, Armesto V, et al. Neovascularization around the optic nerve in giant cell arteritis. Arthritis Rheum. 2003;49:737-738.

39. Geiger J, Ness T, Uhl M, et al. Involvement of the ophthalmic artery in giant cell arteritis visualized by 3T MRI. Rheumatology (Oxford). 2009;48:537-541.

40. Mossa-Basha M, Hwang WD, De Havenon A, et al. Multicontrast high-resolution vessel wall magnetic resonance imaging and its value in differentiating intracrania vasculopathic processes. Stroke. 2015;46:1567-1573. 